\title{
Detection of BRAF V600 Mutations in
} Melanoma: Evaluation of Concordance between the Cobas $^{\circledR} 4800$ BRAF V600 Mutation Test and the Methods Used in French National Cancer Institute (INCa) Platforms in a Real-Life Setting

\author{
Samia Mourah ${ }^{1,2 *}$, Marc G. Denis ${ }^{3}$, Fabienne Escande Narducci ${ }^{4}$, Jérôme Solassol ${ }^{5}$, Jean- \\ Louis Merlin ${ }^{6}$, Jean-Christophe Sabourin ${ }^{7}$, Jean-Yves Scoazec ${ }^{8,9}$, L'Houcine Ouafik $^{10}$, \\ Jean-François Emile ${ }^{11}$, Remy Heller ${ }^{12}$, Claude Souvignet ${ }^{13}$, Loïc Bergougnoux ${ }^{13}$, Jean- \\ Philippe Merlio ${ }^{14}$
}

1 Department of Pharmacology-Genetics, AP-HP, Saint-Louis Hospital, Paris, France, 2 INSERM UMRS 976 Paris, F-75010, France, 3 Department of Biochemistry, Nantes University Hospital, Nantes, France, 4 Department of Molecular Biology and Biochemistry, Lille Regional University Hospital, Lille, France, $\mathbf{5}$ Department of Biology—Pathology, Montpellier University Hospital, Montpellier, France, 6 Department of Tumor Biology, Alexis Vautrin Center, Vandoeuvre les Nancy, France, 7 Department of PathologyCytopathology, Rouen University Hospital, Rouen, France, 8 Laboratory of Pathology and Cytopathology, Edouard Herriot Hospital, Lyon, France, 9 Department of Biology and Pathology, Gustave Roussy Hospital, Villejuif, France, 10 Department of Cancer Biology, Marseille Nord University Hospital, Marseille, France, 11 Department of Pathology, Ambroise Paré Hospital, Boulogne-Billancourt, France, 12 Department of Microbiology and Molecular Biology, Colmar hospital, Colmar, France, 13 Roche, Boulogne-Billancourt, France, 14 Department of Tumor Biology, Bordeaux University Hospital, Bordeaux, France

* samia.mourah@sls.aphp.fr

Academic Editor: John Matthew Koomen, Moffitt Cancer Center, UNITED STATES

Received: July 21, 2014

Accepted: January 20, 2015

Published: March 19, 2015

Copyright: @ 2015 Mourah et al. This is an open access article distributed under the terms of the Creative Commons Attribution License, which permits unrestricted use, distribution, and reproduction in any medium, provided the original author and source are credited.

Data Availability Statement: All relevant data are within the paper.

Funding: Roche - France and INCa for funding tests. The funders participated in study design, data collection and analysis, decision to publish, or preparation of the manuscript.

Competing Interests: The authors have read the journal's policy and have the following competing interests: SM: Scientific committee board

\section{Abstract}

Vemurafenib is approved for the treatment of metastatic melanoma in patients with BRAF V600 mutation. In pivotal clinical trials, BRAF testing has always been done with the approved cobas 4800 BRAF test. In routine practice, several methods are available and are used according to the laboratories usual procedures. A national, multicenter, non-interventional study was conducted with prospective and consecutive collection of tumor samples. A parallel evaluation was performed in routine practice between the cobas $4800 B R A F$ V600 mutation test and home brew methods (HBMs) of 12 national laboratories, labelled and funded by the French National Cancer Institute (INCa). For 420 melanoma samples tested, the cobas method versus HBM showed a high concordance $(93.3 \%$; kappa $=0.86)$ in BRAF V 600 genotyping with similar mutation rates (34.0\% versus $35.7 \%$, respectively). Overall, $97.4 \%$ and $98.6 \%$ of samples gave valid results using the cobas and HBM, respectively. Of the 185 samples strictly fulfilling the cobas guidelines, the concordance rate was even higher $(95.7 \%$; kappa $=0.91 ; 95 \% \mathrm{Cl}[0.85 ; 0.97])$. Out of the 420 samples tested, 28 $(6.7 \%)$ showed discordance between HBM and cobas. This prospective study shows a high 
membership Paraf in study. Consulting fees or other remuneration: Roche, Novartis, GSK. MD: Scientific committee board membership Paraf'in study.

Consulting fees or other remuneration: Roche, AstraZeneca, Boehringer Ingelheim, Qiagen. JLM: Consulting fees or other remuneration, Roche; Other, amgen, bayer, GSK, Lilly, Merck-Serono, Novartis, Roche, Sanofi. JCS: Consulting fees or other remuneration, Roche, Boehringer Ingelmen, Merck; Research grants, Roche; Other, Amgen. JYS: Research grants, Novartis, Ipsen; Consulting fees or other remuneration, Roche. FEN, JS, LHO: none. JFE: Research grants, Roche; Consulting fees or other remuneration, Roche. RH: Research grants, Roche. LB, CS: Employee, Roche. JPM: Scientific committee board membership Paraf in study. This does not alter the authors' adherence to PLOS ONE policies on sharing data and materials. concordance rate between the cobas 4800 BRAF V600 test and home brew methods in the routine detection of BRAF V600E mutations.

\section{Introduction}

The incidence and mortality rates of melanoma have risen sharply throughout the world over the past few decades and the incidence of melanoma has shown the largest increase of all cancers [1]. Cutaneous melanoma is the most serious skin cancer due to its high potential for metastasis[2,3] and, until recently, no effective treatments were available [4].

Recent discoveries in cell signaling pathways that control cellular proliferation have provided a greater understanding of the biology that underlies melanoma and have elucidated the central role of $B R A F$ kinase [5,6]. The mitogen-activated protein kinase (MAPK) pathway is a key regulator of melanoma proliferation and is critical to oncogenic signalling in the majority of patients with cutaneous melanoma. Activating BRAF V600 mutations have been shown to occur in $40 \%-60 \%$ of malignant melanomas $[7,8]$, including in recent reports based on analyses of French patients $[9,10]$.

The discovery of such somatic mutations in the BRAF gene has paved the way for developing targeted therapies in melanoma [11,12]. Indeed, the importance of targeting this pathway for melanoma treatment using specific $B R A F$ inhibitors has been successfully demonstrated in $B R A F$ V600-mutated melanoma in preclinical models $[13,14]$ and, more importantly, in clinical trials [15-18].

Vemurafenib (Zelboraf), a selective BRAF inhibitor, has been shown to increase the overall median survival by 3.6 months (13.2 months in the vemurafenib arm versus 9.6 months in the dacarbazine arm; HR, $0.37 ; 95 \% \mathrm{CI}, 0.26$ to 0.55 ) [15] and has recently been approved as a first line therapy in BRAF (V600E in the USA, V600 in Europe) mutated advanced melanoma $[15,19]$.

Vemurafenib was granted Marketing Authorization (MA) in Europe in February 2012 for the treatment of adult patients with $B R A F$ V600 mutation-positive unresectable or metastatic melanoma. The approval of vemurafenib has made BRAF V600 molecular genotyping mandatory requiring molecular diagnostic testing in order to select patients who will benefit from this therapy [15]. Therefore, vemurafenib was developed conjointly with the cobas $4800 B R A F$ V600 Mutation Test (Roche Molecular Diagnostics) using allele-specific real-time polymerase chain reaction (PCR) and TaqMelt technology to determine BRAF V600 mutation status in DNA isolated from formalin-fixed, paraffin-embedded (FFPE) tumor tissue [20]. It was designed to detect the predominant BRAF V600E mutation with high sensitivity (less than $5 \%$ of V600E sequence in a wild-type sequencing environment).

In August 2011, the cobas 4800 BRAF V600 Mutation Test reagent obtained European Community-In Vitro Diagnostic (EC-IVD) labeling for the detection of the main BRAF V600 somatic mutations in routine diagnostic testing. The analytical performances of the reagent have been evaluated in several multicenter studies [21-23].

In France, the French National Cancer Institute (INCa) has set up a national network of 28 regional molecular cancer genetics platforms where selective molecular tests, including $B R A F$ V600 genotyping, are routinely performed using methods specific to each laboratory [24,25]. In this real-life study, we evaluated the concordance of the cobas 4800 BRAF V600 Mutation Test relative to the home brew methods (HBM) used at 12 participating INCa platforms when tested in parallel for BRAF genotyping in melanoma samples. 


\section{Materials and Methods Melanoma samples}

This national, multicenter, prospective, non-interventional study included 420 consecutive tumor samples of histologically proven melanoma tumor tissue, surgical specimens or biopsies of primary tumors or metastases (regardless of disease stage), fixed and paraffin-embedded. Tumor samples for which the fixative was unknown were excluded and no sample could be included in the study more than once. At selection, 12 INCa platform laboratories equipped with the cobas 4800 System and routinely performing BRAF V600 mutation testing were invited to participate to the study and eligible tumor samples were consecutively included. The study was conducted in accordance with the recommendations of the professional code of ethics and good epidemiological practices established by the Association of French-speaking Epidemiologists [26]. The solicited Ethics Committee (Comité de Protection des Personnes/CPP) considered that neither patient consent nor CPP approval was required for this non-interventional study. Patients data was anonymized during the conduct of the research in an e-CRF. The BRAF mutation testing was done first using the routine method and secondarily using cobas 4800 automated test for the purpose of the study. The samples dedicated to cobas test were anonymized with a patient code. Data analysis performed by the investigator was completely anonymized. The investigator was not the testing operator. Operators performed the test independently of the investigator.

\section{BRAF V600 Detection Methods}

$B R A F$ mutation testing was carried out in parallel by the cobas $4800 B R A F$ V600 Mutation Test (Roche Molecular Diagnostics) and the HBMs routinely used by the 12 participating INCa molecular genetics platforms in French hospitals (Boulogne-Billancourt, Colmar, Lille, Lyon, Marseille, Montpellier, Nantes, Paris, Pessac, Rouen, Vandoeuvre les Nancy and Villejuif). The HBMs included direct Sanger sequencing, pyrosequencing [27], allele-specific PCR [10], SNaPshot and high-resolution melting (HRM) analysis followed by Sanger sequencing in cases with abnormal curve [9]. DNA extraction was performed using either manual or automated registered techniques.

$B R A F$ mutation testing with the cobas 4800 BRAF V600 Mutation Test was performed by personnel trained in the techniques of PCR and the use of the cobas 4800 System in each center and following the procedures in the Package Insert and in the cobas 4800 System Operator's

Manual. The manufacturer's protocol included another DNA extraction starting from the same tumor sample (mainly FFPE sections), also according to the supplier's recommendations. The cobas 4800 BRAF V600 Mutation Test has been optimized and validated for formalinfixed paraffin embedded (FFPE) tissues. Other cobas criteria include formaldehyde fixation, slice thickness $\geq 5 \mu \mathrm{m}$, dewaxing, percentage of tumor cells $\geq 50 \%$ and DNA concentration $\geq 5 \mathrm{ng} / \mu \mathrm{L}$. However, as the study evaluated the concordance in routine practice, the cobas method was also applied to other types of material with different fixatives (as shown in Fig. 1).

In case of discordance between the local HBM technique and the cobas method, the molecular biologist was responsible for making the decision to either make a cross-over between the two samples of extracted DNA and to reanalyze them by the two different techniques or to perform another analysis (retesting, immunohistochemistry for BRAF V600E, etc) according to his/her usual practices. 


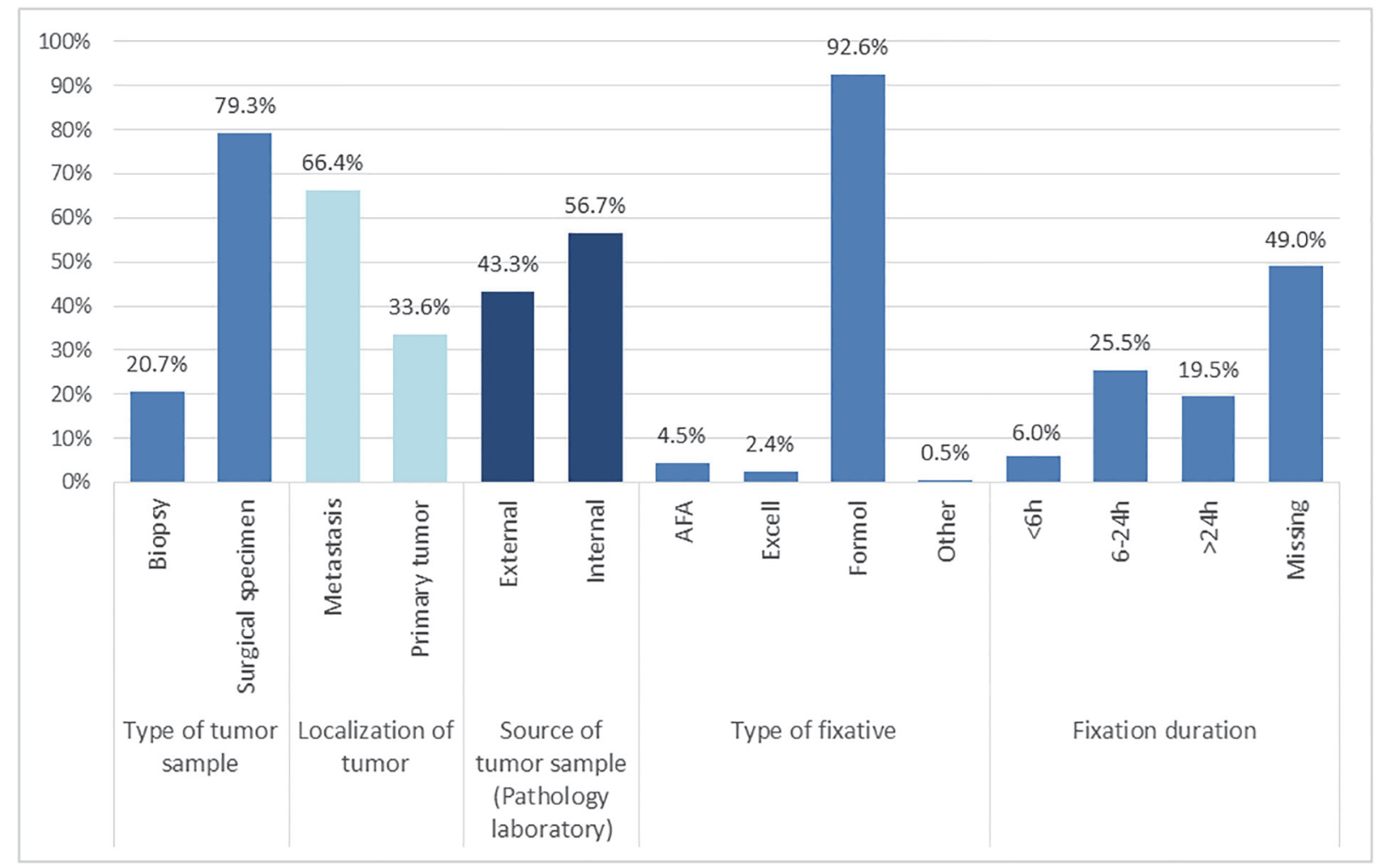

Fig 1. Tumor sample characteristics and method of fixation.

doi:10.1371/journal.pone.0120232.g001

\section{Statistical Analysis}

The primary study objective was to assess the concordance between the cobas 4800 BRAF V600 mutation test and the HBMs for detection of BRAF V600 mutations in melanoma. The primary endpoint was the discordance rate between the INCa molecular laboratory HBM and the cobas 4800 BRAF V600 mutation test for detection of BRAF V600 mutations in melanoma. Secondary endpoints were tumor sample characteristics, descriptions of preanalytical and analytical methods, and management of discordant cases. No changes in the analyses were implemented after protocol signature. The scientific committee requested several additional exploratory analyses, including description of DNA in quantitative amounts and qualitative classes, and description of the preanalytical and analytical methods according to the BRAF V600 testing results.

A total of 384 tumor samples would allow to detect a rate of $10 \%$ of discordance between the two methods with a precision of $3 \%(\alpha=5 \%)$ for the $95 \%$ confidence interval (CI). The total number of samples to be included in this study was 400 , assuming a $5 \%$ rate of non-evaluable samples (damaged equipment, too few tumor cells, etc).

The principal analysis was carried out on the population of evaluable samples. Initially, total mutation rates for the routine methods of INCa molecular genetics platform laboratories and for the cobas 4800 BRAF V600 Mutation Test were described as "V600 mutation", "No V600 mutation" or "Invalid result" (defined as samples that were evaluable but the results could not be interpreted). A discordance was defined when the result of the usual laboratory method was different from that of the cobas 4800 BRAF V600 Mutation Test. The Kappa coefficient was presented with its $95 \%$ CI to evaluate the concordance between the two methods. 
A sensitivity analysis was carried out by not taking into account samples giving "invalid results" for at least one of the two methods. Another sensitivity analysis was performed on the subgroup of samples strictly respecting cobas criteria. A two-sided type one error of 0.05 was used for all statistical tests. As planned in the protocol, an intermediate analysis was performed that was not considered in this paper. Due to the descriptive nature of the statistical analysis, the intermediate analysis did not affect the final analysis. No adjustment of the type one error was needed.

\section{Results}

\section{Melanoma samples}

Between December 2012 and April 2013, 420 melanoma samples were consecutively included in this BRAF V600 mutation parallel testing study conducted in 12 platform laboratories, with a median number of 32 samples (min 20-max 52) in each laboratory. Most tumor samples were surgical specimens $(79 \%)$ and $66 \%$ were samples of metastases. Tumor specimens were provided by the hospital internal pathology laboratory in $57 \%$ of the cases and from other laboratories in the remaining cases (Fig. 1).

\section{Pre-analysis of tumor samples}

The fixation was performed and controlled by the internal pathology laboratory for $56.4 \%$ of the samples (Fig. 1). The time from sampling to fixation (ischemia) was less than 2 hours (33.3\% of all samples) and duration of fixation was $6-24$ hours ( $25.5 \%$ of all samples). The overwhelming majority of samples were formalin-fixed (92.6\%) but three centers also used Alcohol-Formol-Acetic acid and two centers also used Excell Plus for fixation.

Most of the samples (90.7\%) were subjected to paraffin removal prior to DNA extraction. No necrosis was detected in $74.2 \%$ of the samples. The median percentage of tumor cells was $75 \%$ and there were $\geq 50 \%$ of tumor cells in $91.9 \%$ of the samples. Melanin was absent from $51.7 \%$ of the samples and was present in moderate to high amounts in $15.9 \%$ (Table 1).

DNA extraction was performed manually for $55.2 \%$ of the samples using silica-membranebased nucleic acid purification kits (Table 1$)$. The remaining extractions $(\mathrm{n}=188)$ were performed using automated methods. The instruments used were Qiacube (Qiagen) for 79 samples (42.0\%), iPrep (Invitrogen) for 50 (26.6\%), Maxwell 16 (Promega) for 30 (16.0\%), and Qiasymphony (Qiagen) for 29 (15.4\%). Overall, for the HBM, the amount of DNA obtained was at least $5000 \mathrm{ng}$ in $57.7 \%$ of the samples and the median amount of DNA was $6850 \mathrm{ng}$. At least $5000 \mathrm{ng}$ of DNA were obtained in $50.6 \%$ of the samples with automated extraction and in $62.5 \%$ of the samples with manual extraction. The median amount of DNA was $5175 \mathrm{ng}$ and $8234 \mathrm{ng}$, for automated and manual extraction methods respectively.

\section{Description of the analytical methods used in routine practice}

The most frequently used HBM BRAF tests, each used in three centers, were real-time allelespecific PCR (36.7\% of all samples), High Resolution Melting analysis combined with Sanger sequencing $(28.1 \%)$ and pyrosequencing $(16.0 \%)$ (Table 2). Overall, the median size of amplicons was $120 \mathrm{bp}$ [70-232 bp] (Table 2).

A punch was used in four HBM centers (27.9\% of all samples) and was used for $10.7 \%$ of the samples with the cobas test. For both methods, when there was no punch, one to three sections were used in most cases, with a maximum of 10 sections.

The median amount of DNA obtained with the cobas extraction kit was $3635 \mathrm{ng}$ ( $\min 7 \mathrm{ng}-$ $\max 58000 \mathrm{ng})$. 
Table 1. Pre-analytical sample characteristics overall and according to BRAF mutation.

\begin{tabular}{|c|c|c|c|c|c|}
\hline & OVERALLN $=420$ & $\begin{array}{l}\text { BRAF V600 } \\
\text { mutationN = } 136\end{array}$ & $\begin{array}{l}\text { No BRAF V600 } \\
\text { mutationN }=253\end{array}$ & $\begin{array}{l}\text { Discordant } \\
\text { resultN = } 28\end{array}$ & $\begin{array}{l}\text { Invalid } \\
\text { results } \mathrm{N}=3\end{array}$ \\
\hline Slice thickness, $N$ & 386 & 126 & 230 & 27 & 3 \\
\hline Median (min-max) & $5(2-20)$ & $5(3-20)$ & $5(2-20)$ & $4(3-10)$ & $5(4-20)$ \\
\hline$<5 \mu \mathrm{m}$ & $121(31.3 \%)$ & $36(28.6 \%)$ & $69(30.0 \%)$ & $15(55.6 \%)$ & $1(33.3 \%)$ \\
\hline$\geq 5 \mu \mathrm{m}$ & $265(68.7 \%)$ & $90(71.4 \%)$ & $161(70.0 \%)$ & $12(44.4 \%)$ & $2(66.7 \%)$ \\
\hline \multicolumn{6}{|l|}{ Percentage of tumor cells } \\
\hline Median (min-max) & $75(5-100)$ & $70(15-100)$ & $80(5-100)$ & $80(20-100)$ & $90(80-90)$ \\
\hline$<50 \%$ & $34(8.1 \%)$ & $13(9.6 \%)$ & $17(6.7 \%)$ & $4(14.3 \%)$ & 0 \\
\hline$\geq 50 \%$ & $386(91.9 \%)$ & $123(90.4 \%)$ & $236(93.3 \%)$ & $24(85.7 \%)$ & $3(100 \%)$ \\
\hline \multicolumn{6}{|l|}{ DNA extraction method } \\
\hline Automated & $188(44.8 \%)$ & $57(41.9 \%)$ & $110(43.5 \%)$ & $19(67.9 \%)$ & $2(66.7 \%)$ \\
\hline Manual & $232(55.2 \%)$ & $79(58.1 \%)$ & $143(56.5 \%)$ & $9(32.1 \%)$ & $1(33.3 \%)$ \\
\hline Percentage of necrosis, $N$ & 341 & 113 & 210 & 16 & 2 \\
\hline Median (min-max) & $0(0-60)$ & $0(0-50)$ & $0(0-60)$ & $0(0-20)$ & $0(0-0)$ \\
\hline $0 \%$ & $253(74.2 \%)$ & $77(68.1 \%)$ & $164(78.1 \%)$ & $10(62.5 \%)$ & $2(100.0 \%)$ \\
\hline$\geq 0 \%$ & $88(25.8 \%)$ & $36(31.9 \%)$ & $46(21.9 \%)$ & $6(37.5 \%)$ & 0 \\
\hline Presence of melanin, $N$ & 391 & 7 & 12 & 9 & 1 \\
\hline Absent & $202(51.7 \%)$ & $68(52.7 \%)$ & $122(50.6 \%)$ & $11(57.9 \%)$ & $1(50.0 \%)$ \\
\hline Low & $127(32.5 \%)$ & $41(31.8 \%)$ & $80(33.2 \%)$ & $6(31.6 \%)$ & 0 \\
\hline Medium & $43(11.0 \%)$ & $15(11.6 \%)$ & $26(10.8 \%)$ & $1(5.3 \%)$ & $1(50.0 \%)$ \\
\hline High & $19(4.9 \%)$ & $5(3.9 \%)$ & $13(5.4 \%)$ & $1(5.3 \%)$ & 0 \\
\hline \multicolumn{6}{|l|}{ DNA elution volume $(\mu \mathrm{L})$} \\
\hline Median (min-max) & $100(25-185)$ & $100(25-185)$ & $100(25-185)$ & $100(25-185)$ & $60(50-100)$ \\
\hline $\begin{array}{l}\text { DNA concentration } \\
\text { (ng/pL), } N\end{array}$ & 392 & 130 & 233 & 26 & 3 \\
\hline \multirow[t]{2}{*}{ Median (min-max) } & 76.5 & 89.0 & 75.0 & 32.3 & 4.0 \\
\hline & $(0.6-1514)$ & $(0.6-1514)$ & $(0.6-1465)$ & $(1.3-468)$ & $(1.5-54)$ \\
\hline$<5 \mathrm{ng} / \mu \mathrm{L}$ & $36(9.2 \%)$ & $10(7.7 \%)$ & $18(7.7 \%)$ & $6(23.1 \%)$ & $2(66.7 \%)$ \\
\hline$\geq 5 \mathrm{ng} / \mu \mathrm{L}$ & $356(90.8 \%)$ & $120(92.3 \%)$ & $215(92.3 \%)$ & 20 (76.9\%) & $1(33.3 \%)$ \\
\hline $\begin{array}{l}\text { Amount of DNA for HBM } \\
\text { (ng) } N\end{array}$ & 392 & 130 & 233 & 26 & 3 \\
\hline \multirow[t]{2}{*}{ Median (min-max) } & 6850 & 7800 & 6375 & 3235 & 240 \\
\hline & $(15-102200)$ & $(15-102200)$ & $(15-75500)$ & $(33-37206)$ & $(146-2700)$ \\
\hline$<5000$ ng & $166(42.3 \%)$ & $50(38.5 \%)$ & 99 (42.5\%) & $14(53.8 \%)$ & $3(100 \%)$ \\
\hline$\geq 5000 \mathrm{ng}$ & $226(57.7 \%)$ & $80(61.5 \%)$ & $134(57.5 \%)$ & $12(46.2 \%)$ & 0 \\
\hline
\end{tabular}

doi:10.1371/journal.pone.0120232.t001

\section{Concordance between cobas and HBM}

The frequency of BRAF V600 mutations detected were $34.0 \%$ with the cobas vs $35.7 \%$ with the HBM methods (Table 3). Wild-type genotypes were observed in $63.3 \%$ and $62.9 \%$ of the samples using cobas and HBM tests respectively. Overall, the genotyping tests delivered a valid result (either presence or absence of BRAF mutation) in $97.3 \%$ of tumors with the cobas and 98.6\% of tumors with HBM tests respectively.

A high concordance rate of 93.3\% (392 samples) and a kappa coefficient of 0.86 (95\% CI $[0.81 ; 0.91])$ were obtained on comparing cobas and HBM methods for BRAF V600 mutation detection in routine practice (Table 3 -all samples). The concordance and kappa coefficient were even better when taking into account the 389 evaluables samples (concordance 
Table 2. Analytical method sample characteristics overall and according to BRAF mutation.

\begin{tabular}{|c|c|c|c|c|c|}
\hline & OVERALLN = 420 & $\begin{array}{l}\text { BRAF V600 } \\
\text { mutationN = } 136\end{array}$ & $\begin{array}{l}\text { No BRAF V600 } \\
\text { mutationN }=253\end{array}$ & $\begin{array}{l}\text { Discordant } \\
\text { resultN = } 28\end{array}$ & Indeterminate $\mathrm{N}=3$ \\
\hline \multicolumn{6}{|c|}{ HOME BREW ANALYTICAL METHOD } \\
\hline \multicolumn{6}{|l|}{$\begin{array}{l}\text { Method of mutation } \\
\text { detection }\end{array}$} \\
\hline Allele-specific/real time PCR & $154(36.7 \%)$ & $47(34.5 \%)$ & $100(39.5 \%)$ & $7(25.0 \%)$ & 0 \\
\hline HRM + Sanger sequencing & $118(28.1 \%)$ & $36(26.5 \%)$ & $73(28.9 \%)$ & $8(28.6 \%)$ & $1(33.3 \%)$ \\
\hline Pyrosequencing & $67(16.0 \%)$ & $28(20.6 \%)$ & $37(14.6 \%)$ & $1(3.6 \%)$ & $1(33.3 \%)$ \\
\hline SNaPshot & $30(7.1 \%)$ & $11(8.1 \%)$ & $19(7.5 \%)$ & 0 & 0 \\
\hline Sanger sequencing & $51(12.1 \%)$ & $14(10.3 \%)$ & $24(9.5 \%)$ & $12(42.9 \%)$ & $1(33.3 \%)$ \\
\hline \multicolumn{6}{|l|}{$\begin{array}{l}\text { Size of amplicons used } \\
\text { (base pairs [bp]) }\end{array}$} \\
\hline Median (min-max) & $120(70-232)$ & $120(70-232)$ & $120(70-232)$ & $157(70-232)$ & $107(82-221)$ \\
\hline \multicolumn{6}{|c|}{ COBAS ANALYTICAL METHOD } \\
\hline Amount of DNA (ng) $N$ & 420 & 136 & 253 & 28 & 3 \\
\hline \multirow[t]{2}{*}{ Median (min-max) } & 3635 & 4680 & 3350 & 4085 & 467 \\
\hline & $(7-58000)$ & $(7-30000)$ & $(300-58000)$ & $(300-49000)$ & $(450-782)$ \\
\hline$<5000 n g$ & $250(59.5 \%)$ & $69(50.7 \%)$ & $162(64.0 \%)$ & $16(57.1 \%)$ & $3(100 \%)$ \\
\hline$\geq 5000 \mathrm{ng}$ & $170(40.5 \%)$ & 67 (49.3\%) & $91(36.0 \%)$ & $12(42.9 \%)$ & 0 \\
\hline
\end{tabular}

doi:10.1371/journal.pone.0120232.t002

rate $=95.8 \%$, kappa $=0.91$ and when taking into account the 185 samples strictly respecting the cobas criteria (concordance rate $=95,7 \%$, kappa $=0.91$ ).

With HBM, the main types of mutation detected were V600E in $88.7 \%$ of the mutations and V600K in 9.3\%, others included V600R (1.3\%) and V600D (0.7\%). Six samples (1.4\%) gave invalid results with the in-house method and 11 (2.6\%) with the cobas test.

A further analysis performed taking into account the 185 samples strictly respecting the cobas criteria gave a higher concordance rate of $95.7 \%$ and a kappa coefficient of 0.91 (95\% CI $[0.85 ; 0.97]$ ) (Table 3 - samples strictly respecting cobas criteria).

\section{Analysis of the discordant cases}

Results from the cobas method and the HBM were discordant for $28 / 420$ samples (6.7\%) (Table 3 -all samples). Among these 28 discordant cases, 11 had invalid results (8 samples with the cobas and 3 with HBM) and 17 were true discordant cases ( 7 with BRAF V600 mutation detected by the cobas only and 10 detected by HBM only). For the 10 samples with BRAF V600 mutation detected by the HBM but not by the cobas Mutation Test. Five of them were V600K, D or R mutations. Of these 5 cases, all specimens contained $\geq 50 \%$ of tumor cells, except one ( $40 \%$ of tumor cells). Of the other 5 samples with V600E mutation not detected by the cobas Mutation Test, two of the specimens contained only 20\% tumor cells. For the 7 other samples with BRAF V600 mutation detected by the cobas' but not by HBMs, this result was confirmed after retesting in 4 samples, the other samples were considered as having invalid results $(\mathrm{n}=2)$ or not mutated $(\mathrm{n}=1)$.

Univariate logistic regression analyses performed on tumor samples for which there was discordance between the two methods showed that explanatory factors for discordance may be Sanger sequencing $(\mathrm{p}<0.0001)$, slice thickness $(>5 \mu \mathrm{m} \mathrm{p}=0.0068)$ and automated DNA extraction $(\mathrm{p}=0.014)$ (Table 4$)$. Multivariate analysis showed that automated DNA extraction method $(\mathrm{p}=0.0411)$ and Sanger sequencing method $(\mathrm{p}<0.0001)$ could be two potential explanatory factors of discordance (Table 4). 
Table 3. Description of concordances and discordances between the cobas method and home brew methods (all samples $\mathrm{N}=420$ and samples strictly respecting cobas criteria $\mathrm{N}=185$ ).

\begin{tabular}{|c|c|c|c|c|}
\hline \multirow{2}{*}{$\begin{array}{l}\text { All samples } \\
\text { Home brew methods }\end{array}$} & \multicolumn{4}{|c|}{ Cobas method } \\
\hline & BRAF V600 mutation & No BRAF V600 mutation & Invalid results & $\begin{array}{l}\text { Total } \\
\mathrm{N}=420\end{array}$ \\
\hline BRAF V600 mutation & $136(32.38 \%)$ & $10(2.38 \%)$ & $4(0.95 \%)$ & $150(35.7 \%)$ \\
\hline No BRAF V600 mutation & $7(1.67 \%)$ & $253(60.24 \%)$ & $4(0.95 \%)$ & $264(62.9 \%)$ \\
\hline Invalid results & - & $3(0.71 \%)$ & $3(0.71 \%)$ & $6(1.4 \%)$ \\
\hline Total & $143(34.0 \%)$ & $266(63.3 \%)$ & $11(2.6 \%)$ & \\
\hline n (\%) concordant & $392(93.3 \%)$ & & & \\
\hline n (\%) discordant & \multicolumn{4}{|c|}{28 (6.7\%) 95\% Cl: [4.48\%; 9.49\%] } \\
\hline Kappa coefficient & \multicolumn{4}{|c|}{0.8611 95\% Cl: [0.8125; 0.9097] } \\
\hline Samples strictly respecting cobas criteria & \multicolumn{4}{|l|}{ Cobas method } \\
\hline Home brew methods & BRAF V600 mutation & No BRAF V600 mutation & Invalid results & $\begin{array}{l}\text { Total } \\
N=185\end{array}$ \\
\hline BRAF V600 mutation & $63(34.05 \%)$ & $1(0.54 \%)$ & $1(0.54 \%)$ & $65(35.1 \%)$ \\
\hline No BRAF V600 mutation & $3(1.62 \%)$ & $114(61.62 \%)$ & $2(1.08 \%)$ & $119(64.3 \%)$ \\
\hline Invalid results & - & $1(0.54 \%)$ & - & $1(0.5 \%)$ \\
\hline Total & $66(35.7 \%)$ & $116(62.7 \%)$ & $3(1.6 \%)$ & \\
\hline n (\%) concordant & \multicolumn{4}{|l|}{177 (95.7\%) } \\
\hline n (\%) discordant & \multicolumn{4}{|c|}{8 (4.3\%) 95\% Cl: [1.89\%; 8.34\%] } \\
\hline Kappa coefficient & \multicolumn{4}{|c|}{0.9082 95\% Cl: [0.8472; 0.9692] } \\
\hline
\end{tabular}

doi:10.1371/journal.pone.0120232.t003

Among the 12 centers participating in this study, the discordance rate ranged from $0 \%$ to $31 \%$. The highest discordance rates (13.6\% and 31.0\%) occurred in two centers using Sanger sequencing and long amplicons (157 and $221 \mathrm{bp})$.

\section{Discussion}

The cobas 4800 BRAF V600 Mutation Test has been developed within the strict framework of clinical trials as a companion diagnostic for targeted cancer therapy with vemurafenib, a BRAF kinase inhibitor [20].

Here we report on the first prospective and multicenter study assessing in routine practice testing of BRAF V600 mutations in melanoma samples by HBM in molecular genetics laboratories in parallel with the cobas test, an approved and automated assay. Results showed a high concordance rate of $93.3 \%$ (Kappa $=0.86$ ) between the two assessments.

The rates of BRAF V600 mutations observed between the large array of different HBM and cobas tests were very close (35.7\% for HBM and $34.0 \%$ for cobas). These results are consistent with the INCa platforms annual report for the year 2012 during which 3,479 patients underwent $B R A F$ testing and the frequency of mutation detection for the $B R A F$ gene was $37.6 \%$ [28]. In the INCa report, mutation frequency varied depending on the mutation detection method from $30.6 \%$ with TaqMan real-time PCR to $42.7 \%$ with allele-specific PCR [28]. Compared to the general situation in INCa platforms, the present study included less laboratories performing direct Sanger sequencing and more tests were performed using allele-specific PCR [29]. Allele-specific PCR, High Resolution Melting analysis combined with Sanger sequencing, or pyrosequencing were used in the centers with the highest annual activity for malignant melanoma testing. 
Table 4. Univariate and multivariate analyses of discordant results.

\begin{tabular}{|c|c|c|c|c|c|}
\hline & \multirow[t]{2}{*}{$\%$ Discordant $^{\S}$} & Univariate analysis & \multirow[t]{2}{*}{$p$-value } & Multivariate analysis & \multirow[t]{2}{*}{$p$-value } \\
\hline & & OR $[95 \% \mathrm{Cl}]$ & & OR $[95 \% \mathrm{Cl}]$ & \\
\hline \multicolumn{6}{|c|}{ PRE-ANALYTICAL METHOD } \\
\hline \multicolumn{6}{|l|}{ Source of tumor sample } \\
\hline External pathology lab & $5.5 \%$ & & & & \\
\hline Internal pathology lab & $7.6 \%$ & $1.407[0.633,3.126]$ & 0.4017 & & \\
\hline \multicolumn{6}{|c|}{ Slice thickness $(\mu \mathrm{m})$ (classes) } \\
\hline$<5 \mu \mathrm{m}$ & $12.4 \%$ & $2.984[1.351,6.589]$ & $0.0068 *$ & & \\
\hline$\geq 5 \mu \mathrm{m}$ & $4.5 \%$ & & & & \\
\hline \multicolumn{6}{|c|}{ Percentage of tumor cells (classes) } \\
\hline$<50 \%$ & $11.8 \%$ & $2.011[0.655,6.177]$ & 0.2223 & & \\
\hline$\geq 50 \%$ & $6.2 \%$ & & & & \\
\hline \multicolumn{6}{|l|}{ Extraction method } \\
\hline Automated & $10.1 \%$ & $2.786[1.229,6.311]$ & $0.0141 *$ & & \\
\hline Manual & $3.9 \%$ & & & $2.487[1.038 ; 5.964]$ & $0.0411 *$ \\
\hline \multicolumn{6}{|c|}{ HOME BREW METHODS } \\
\hline \multicolumn{6}{|c|}{ Amount of DNA (ng)-pre-analytical } \\
\hline$<5000$ ng & $8.4 \%$ & $1.643[0.739,3.650]$ & 0.2232 & & \\
\hline$\geq 5000 \mathrm{ng}$ & $5.3 \%$ & & & & \\
\hline \multicolumn{6}{|l|}{ Sanger sequencing } \\
\hline No & $4.3 \%$ & & & $6.264[2.708 ; 14.487]$ & $<0.0001^{*}$ \\
\hline Yes & $23.5 \%$ & $6.788[2.995,15.387]$ & $<0.0001^{*}$ & & \\
\hline \multicolumn{6}{|l|}{ Punch } \\
\hline No & $6.9 \%$ & $1.170[0.484,2.831]$ & 0.7273 & & \\
\hline Yes & $6.0 \%$ & & & & \\
\hline \multicolumn{6}{|l|}{ COBAS METHOD } \\
\hline \multicolumn{6}{|c|}{ Amount of DNA (ng)-pre-analytical } \\
\hline$<5000 n g$ & $6.4 \%$ & & & & \\
\hline$\geq 5000 \mathrm{ng}$ & $7.1 \%$ & $1.111[0.512,2.412]$ & 0.7906 & & \\
\hline \multicolumn{6}{|l|}{ Punch } \\
\hline No & $6.9 \%$ & $1.602[0.367,6.985]$ & 0.5307 & & \\
\hline Yes & $4.4 \%$ & & & & \\
\hline
\end{tabular}

§Percentages are calculated on the total number of samples $(\mathrm{N}=420)$;

*The most highly significant values.

doi:10.1371/journal.pone.0120232.t004

Overall, the rates of invalid results were low, at $1.4 \%$ and $2.6 \%$ for the HBM and cobas tests respectively, compared to the 5.1\% reported in the INCa report [28]. The INCa report showed a variation in the frequency of invalid results according to the mutation detection method from $2.5 \%$ with allele-specific PCR to $7.0 \%$ with direct Sanger sequencing.

Among the 10 cases of discordant BRAF V600E genotypes, it is difficult to conclude whether this discordance is due to possible contaminations, tumor heterogeneity or to other factors such as DNA quality or preanalytical parameters. The characteristics of the preanalytical and analytical methods were mostly similar for samples whatever the BRAF V600 mutation result (V600 mutation, no V600 mutation, discordant or invalid result). Indeed, any attempts to highlight specific preanalytical parameters to explain the discordance are hindered as ischemia and fixation time parameters were missing for about half of the samples. Also, potential 
contaminations during automated DNA extraction, although unlikely, cannot be excluded. Intra-tumoral heterogeneity should also be taken into account. Indeed, using laser microdissection followed by mutation specific Snapshot assay, a substantial proportion of individual tumor specimens were found to contain a mixture of BRAF mutant and wild-type melanoma cells [30]. In addition, a low percentage of tumor cells may possibly explain one case of discordance according to the molecular technique sensitivity threshold.

As expected, based on preclinical and clinical studies involving retrospective sequencing, rare V600R, V600D and V600K mutations were detected with low sensitivity by cobas [22,31,32]. In a study of 295 melanoma samples comparing the cobas 4800 BRAF V600 Mutation Test with direct Sanger sequencing assays, the cobas test was less sensitive for mutations other than the single-nucleotide V600E mutation [33]. In this retrospective analysis of BRAF V600 mutations, twenty samples were excluded because of invalid results (by cobas $(n=3)$, sequencing $(\mathrm{n}=15)$, or both $(\mathrm{n}=2))$ and 23 samples with positive sequencing results gave negative cobas results [33].

Tumors with other V600 mutations, such as V600K, are sensitive to vemurafenib treatment [34]. In our study, only 2 discordant samples had V600K mutations, as detected by HBM but not with cobas.

Concerning the analytical genotyping method used, a higher discordance rate was observed in centers using direct Sanger sequencing with long amplicons for mutation detection. Low sensitivity (15-30\%) of the Sanger sequencing method has previously been reported $[22,23,35,36]$. Furthermore, false positive detections by the Sanger method could be due to contamination or background noise [35]. Conversely, high concordance was observed between the cobas method and the High Resolution Melting analysis technique followed by Sanger sequencing.

Interestingly, both pyrosequencing and High Resolution Melting analysis methods have been shown by two French groups participating in this study to provide concordant data with immunohistochemical detection of the BRAF p.V600E protein when applying robust and simple interpretation criteria $[9,27]$. Although rare false negative cases exist using immunohistochemistry as a screening tool, these cases would be tested with DNA-based methods in a hierarchical algorithm [37]. On the other hand, "false positive" cases detected by immunohistochemistry were reported wild type using direct sequencing, but further detected as mutated using Pyrosequencing [37]. Altogether our molecular comparison between cobas and HB methods also indicated that direct sequencing should not be used in routine testing of melanoma samples as well as in technical comparison since this generates a high rate of discordant cases.

In conclusion, the results of this first prospective study comparing the cobas $4800 \mathrm{BRAF}$ V600 Mutation Test and various HBM developed in national platforms in France allows us to draw 2 main conclusions: standard DNA sequencing (especially using long DNA amplicons) generates a significant proportion of invalid results, and the cobas 4800 BRAF V600 mutation assay is efficient for testing V600E BRAF mutations.

\section{Acknowledgments}

The authors wish to thank the participating pathologists who sent the samples to the platforms as well as participating oncodermatologists.

The authors also wish to thank Samuel Gally (project management), Sandrine Kraemer and Mathieu Coudert (statistical analysis) and Christine Laurent (data management).

Medical writing assistance and editing was provided by Eltium (France). 


\section{Author Contributions}

Conceived and designed the experiments: SM MD JCS JYS JPM LHO. Performed the experiments: SM MD FEN JS JLM JCS JYS LHO JFE RH CS LB JPM. Analyzed the data: SM MD FEN JS JLM JCS JYS LHO JFE RH CS LB JPM. Contributed reagents/materials/analysis tools: SM MD FEN JS JLM JCS JYS LHO JFE RH JPM. Wrote the paper: SM MD FEN JS JLM JCS JYS LHO JFE RH CS LB JPM.

\section{References}

1. Siegel R, DeSantis $C$, Virgo $K$, Stein $K$, Mariotto $A$, Smith $T$, et al. Cancer treatment and survivorship statistics, 2012. CA Cancer J Clin 2012, 62(4): 220-241. doi: 10.3322/caac.21149 PMID: 22700443

2. INCa. La situation du cancer en France en 2010. Collection Rapports \& synthèses, ouvrage collectif édité par l'INCa, Boulogne-Billancourt, novembre 2010. [INCa. The situation in France in 2010. Reports and summaries, collective work edited by INCa, Boulogne-Billancourt, November 2010].

3. Forsea AM, Del Marmol V, de Vries E, Bailey EE, Geller AC. Melanoma incidence and mortality in Europe: new estimates, persistent disparities. Br J Dermatol 2012, 167(5): 1124-1130. doi: 10.1111/j. 1365-2133.2012.11125.x PMID: 22759278

4. Gray-Schopfer V, Wellbrock C, Marais R. Melanoma biology and new targeted therapy. Nature 2007, 445(7130): 851-857. PMID: 17314971

5. Davies H, Bignell GR, Cox C, Stephens P, Edkins S, Clegg S, et al. Mutations of the BRAF gene in human cancer. Nature 2002, 417(6892): 949-954. PMID: 12068308

6. Pollock PM, Harper UL, Hansen KS, Yudt LM, Stark M, Robbins CM, et al. High frequency of BRAF mutations in nevi. Nat Genet 2003, 33(1): 19-20. PMID: 12447372

7. Lee JH, Choi JW, Kim YS. Frequencies of BRAF and NRAS mutations are different in histological types and sites of origin of cutaneous melanoma: a meta-analysis. Br J Dermatol 2011, 164(4): 776-784. doi: 10.1111/j.1365-2133.2010.10185.x PMID: 21166657

8. Long GV, Menzies AM, Nagrial AM, Haydu LE, Hamilton AL, Mann GJ, et al. Prognostic and clinicopathologic associations of oncogenic BRAF in metastatic melanoma. J Clin Oncol 2011, 29(10): 12391246. doi: 10.1200/JCO.2010.32.4327 PMID: 21343559

9. Boursault L, Haddad V, Vergier B, Cappellen D, Verdon S, Bellocq JP, et al. Tumor homogeneity between primary and metastatic sites for BRAF status in metastatic melanoma determined by immunohistochemical and molecular testing. PLoS One. 2013 Aug 20; 8(8):e70826. doi: 10.1371/journal.pone. 0070826 PMID: 23976959

10. Saint-Jean M, Quereux G, Nguyen JM, Peuvrel L, Brocard A, Vallee A, et al. Is a Single BRAF WildType Test Sufficient to Exclude Melanoma Patients from Vemurafenib Therapy? J Invest Dermatol 2013, 134(5):1468-1470. doi: 10.1038/jid.2013.378 PMID: 24025553

11. Curtin JA, Fridlyand J, Kageshita T, Patel HN, Busam KJ, Kutzner H, et al. Distinct sets of genetic alterations in melanoma. N Engl J Med 2005, 353(20): 2135-2147. PMID: 16291983

12. Fecher LA, Amaravadi RK, Flaherty KT. The MAPK pathway in melanoma. Curr Opin Oncol 2008, 20 (2): 183-189. doi: 10.1097/CCO.0b013e3282f5271c PMID: 18300768

13. Amaria RN, Lewis KD, Jimeno A. Vemurafenib: the road to personalized medicine in melanoma. Drugs Today (Barc) 2012, 48(2): 109-118. doi: 10.1358/dot.2012.48.2.1745274 PMID: 22384451

14. Flaherty KT, Puzanov I, Kim KB, Ribas A, McArthur GA, Sosman JA, et al. Inhibition of mutated, activated BRAF in metastatic melanoma. N Engl J Med 2010, 363(9): 809-819. doi: 10.1056/ NEJMoa1002011 PMID: 20818844

15. Chapman PB, Hauschild A, Robert C, Haanen JB, Ascierto P, Larkin J, et al. Improved survival with vemurafenib in melanoma with BRAF V600E mutation. N Engl J Med 2011, 364(26): 2507-2516. doi: 10.1056/NEJMoa1103782 PMID: 21639808

16. Tsai J, Lee JT, Wang W, Zhang J, Cho H, Mamo S, et al. Discovery of a selective inhibitor of oncogenic B-Raf kinase with potent antimelanoma activity. Proc Natl Acad Sci U S A 2008, 105(8): 3041-3046. doi: 10.1073/pnas.0711741105 PMID: 18287029

17. Flaherty KT, Robert $C$, Hersey $P$, Nathan $P$, Garbe $C$, Milhem M, et al. Improved survival with MEK inhibition in BRAF-mutated melanoma. N Engl J Med 2012, 367(2): 107-114. doi: 10.1056/ NEJMoa1203421 PMID: 22663011

18. Hauschild A, Grob JJ, Demidov LV, Jouary T, Gutzmer R, Millward M, et al. Dabrafenib in BRAF-mutated metastatic melanoma: a multicentre, open-label, phase 3 randomised controlled trial. Lancet 2012, 380(9839): 358-365. doi: 10.1016/S0140-6736(12)60868-X PMID: 22735384 
19. Sosman JA, Kim KB, Schuchter L, Gonzalez R, Pavlick AC, Weber JS, et al. Survival in BRAF V600mutant advanced melanoma treated with vemurafenib. N Engl J Med 2012, 366(8): 707-714. doi: 10. 1056/NEJMoa1112302 PMID: 22356324

20. Cheng S, Koch WH, Wu L. Co-development of a companion diagnostic for targeted cancer therapy. N Biotechnol 2012, 29(6): 682-688. doi: 10.1016/j.nbt.2012.02.002 PMID: 22391147

21. Anderson S, Bloom KJ, Vallera DU, Rueschoff J, Meldrum C, Schilling R, et al. Multisite analytic performance studies of a real-time polymerase chain reaction assay for the detection of BRAF V600E mutations in formalin-fixed, paraffin-embedded tissue specimens of malignant melanoma. Arch Pathol Lab Med 2012, 136(11): 1385-1391. doi: 10.5858/arpa.2011-0505-OA PMID: 22332713

22. Halait $\mathrm{H}$, Demartin $\mathrm{K}$, Shah $\mathrm{S}$, Soviero $\mathrm{S}$, Langland $\mathrm{R}$, Cheng $\mathrm{S}$, et al. Analytical performance of a realtime PCR-based assay for V600 mutations in the BRAF gene, used as the companion diagnostic test for the novel BRAF inhibitor vemurafenib in metastatic melanoma. Diagn Mol Pathol 2012, 21(1): 1-8. doi: 10.1097/PDM.0b013e31823b216f PMID: 22306669

23. Lade-Keller J, Romer KM, Guldberg P, Riber-Hansen R, Hansen LL, Steiniche T, et al. Evaluation of BRAF mutation testing methodologies in formalin-fixed, paraffin-embedded cutaneous melanomas. $J$ Mol Diagn 2013, 15(1): 70-80. doi: 10.1016/j.jmoldx.2012.08.003 PMID: 23159593

24. Nowak F, Soria JC, Calvo F. Tumour molecular profiling for deciding therapy-the French initiative. Nat Rev Clin Oncol 2012, 9(8): 479-486. doi: 10.1038/nrclinonc.2012.42 PMID: 22777058

25. Nowak F, Calvo F, Soria JC. Europe Does It Better: Molecular Testing across a National Health Care System-The French Example. Am Soc Clin Oncol Educ Book 33(332-7, 2013.

26. Recommandations de Déontologie et bonnes pratiques en épidémiologie. ADELF, 2007. [Code of Deontology and Good Epidemiological Practices].

27. Colomba E, Helias-Rodzewicz Z, Von Deimling A, Marin C, Terrones N, Pechaud D, et al. Detection of BRAF p.V600E mutations in melanomas: comparison of four methods argues for sequential use of immunohistochemistry and pyrosequencing. J Mol Diagn 2013, 15(1): 94-100. doi: 10.1016/j.jmoldx. 2012.09.001 PMID: 23159108

28. INCa. Les tests de génétique moléculaire pour l'accès aux therapies ciblées en 2012. Collection Bilans d'activité et d'évaluation, ouvrage collectif édité par l'INCa, Boulogne-Billancourt, décembre 2012. [INCa.Molecular genetics tests for access to targeted therapies in 2012. Collective summary of activity and evaluation, collective work edited by INCa, Boulogne-Billancourt, December 2012].

29. INCa. Synthèse de l'activité des plateformes hospitalières de génétique moléculaire des cancers en 2011. Collection Bilan d'activité et d'évaluation, ouvrage collectif édité par l'INCa, Boulogne-Billancourt, décembre 2012. [INCa. Review of the activity of hospital platforms of cancer molecular genetics in 2011. Collective summary of activity and evaluation, collective work edited by INCa, Boulogne-Billancourt, December 2012].

30. Yancovitz M, Litterman A, Yoon J, Ng E, Shapiro RL, Berman RS, et al. Intra- and inter-tumor heterogeneity of BRAF(V600E)) mutations in primary and metastatic melanoma. PLoS One 7(1): e29336, 2012. doi: 10.1371/journal.pone.0029336 PMID: 22235286

31. Zelboraf Summary of Product Characteristics.

32. Ihle MA, Fassunke J, Konig K, Grunewald I, Schlaak M, Kreuzberg N, et al. Comparison of high resolution melting analysis, pyrosequencing, next generation sequencing and immunohistochemistry to conventional Sanger sequencing for the detection of p.V600E and non-p.V600E BRAF mutations. BMC Cancer 2014, 14(1): 13.

33. Qu K, Pan Q, Zhang X, Rodriguez L, Zhang K, Li H, et al. Detection of BRAF V600 mutations in metastatic melanoma: comparison of the Cobas 4800 and Sanger sequencing assays. J Mol Diagn 2013, 15(6): 790-795. doi: 10.1016/j.jmoldx.2013.07.003 PMID: 23994118

34. McArthur GA, Chapman PB, Robert C, Larkin J, Haanen JB, Dummer R, et al. Safety and efficacy of vemurafenib in BRAF(V600E) and BRAF(V600K) mutation-positive melanoma (BRIM-3): extended follow-up of a phase 3, randomised, open-label study. Lancet Oncol 2014; 15(3):323-32. doi: 10.1016/ S1470-2045(14)70012-9 PMID: 24508103

35. Curry JL, Torres-Cabala CA, Tetzlaff MT, Bowman C, Prieto VG. Molecular platforms utilized to detect BRAF V600E mutation in melanoma. Semin Cutan Med Surg 2012, 31(4): 267-273. doi: 10.1016/j. sder.2012.07.007 PMID: 23174497

36. Cousins MM, Donnell D, Eshleman SH. Impact of mutation type and amplicon characteristics on genetic diversity measures generated using a high-resolution melting diversity assay. J Mol Diagn 2013, 15 (1): 130-137. doi: 10.1016/j.jmoldx.2012.08.008 PMID: 23178437

37. Skorokhod A, Capper D, von Deimling A, Enk A, Helmbold P. Detection of BRAF V600E mutations in skin metastases of malignant melanoma by monoclonal antibody VE1. J Am Acad Dermatol 2012; 67 (3):488-91. doi: 10.1016/j.jaad.2012.03.022 PMID: 22890732 